\title{
Training of the respiratory muscles in individuals with tetraplegia
}

\author{
SG Uij1 ${ }^{1}$, S Houtman ${ }^{1}$, HThM Folgering ${ }^{2}$ and MTE Hopman*,1 \\ ${ }^{1}$ Department of Physiology, University of Nijmegen, The Netherlands; ${ }^{2}$ Department of Pulmonary Diseases \\ Dekkerswald, University of Nijmegen, The Netherlands
}

\begin{abstract}
Study design: An experimental cross-sectional design.
Objectives: To evaluate whether training of the innervated respiratory muscles in individuals with a (partial) cervical spinal cord injury will improve the strength and endurance capacity of these muscles and the exercise performance in these individuals.

Setting: Department of Physiology and Pulmonary diseases, Nijmegen, The Netherlands.

Method: In this study nine individuals with tetraplegia $(\mathrm{C} 3-\mathrm{C} 7)$ performed a target flow endurance training of the inspiratory muscles, twice a day for $15 \mathrm{~min}$. First, the subjects performed a 'sham' training for 6 weeks with no appreciable resistance, after that they performed a 'real' training for 6 weeks with a resistance of $70 \%$ of the maximal endurance capacity of the inspiratory muscles. The training was evaluated at 0,6 and 12 weeks by the following tests: (1) the slow Inspiratory Vital Capacity (IVC) and the Forced Inspiratory and Expiratory Volumes over $1 \mathrm{~s}\left(\mathrm{FIV}_{1}\right.$ and $\left.\mathrm{FEV}_{1}\right) ;(2)$ the Maximal Inspiratory Mouth Pressure $\left(\mathrm{P}_{\text {imax }}\right)$ and the Endurance Pressure $\left(\mathrm{P}_{\text {endu }}\right)$ and (3) a maximal arm-cranking exercise test.

Results: After the sham training, the $\mathrm{P}_{\text {endu }}$ was increased from 3.98 to $4.71 \mathrm{kPa}$ with a $P$ value of 0.05 . The sham training had no influence on any of the other variables. The real training had no effect on the IVC, FIV, $\mathrm{FEV}_{1}$ and $\mathrm{P}_{\text {imax }}$, however, increased the $\mathrm{P}_{\text {endu }}$ from 4.71 to $6.16 \mathrm{kPa}(P=0.01)$, representing the respiratory muscle-endurance capacity. The oxygen consumption ( $\mathrm{V}_{2}$ peak) in the maximal exercise test improved from 0.87 to $0.98 \mathrm{l} /$ $\min (P=0.05)$.

Conclusion: The results of the study indicate that training of the respiratory muscles results in an enhanced endurance capacity of these muscles and a concomitant increase in the aerobic exercise performance.
\end{abstract}

Keywords: spinal cord injury; lung function; target flow training; arm cranking exercise; muscle strength and endurance

\section{Introduction}

In individuals with cervical lesions below $\mathrm{C} 4$, the motor innervation of the diaphragm and the sternocleidomastoid muscle will be intact, so one can breathe independently. The loss of innervation depends on the level and completeness of the lesion. This induces another, most likely less efficiënt, breathing pattern in individuals with complete as well as incomplete lesions. In individuals with tetraplegia the lung volume is changed, particularly the Vital Capacity (VC) is decreased. $^{1-4}$ The decrease in $\mathrm{VC}$ is mostly induced by a decrease in Expiratory Reserve Capacity (ERC) and an increase in Residual Volume (RV).,3 The Maximal Expiratory Flow is also decreased. ${ }^{2}$ Denervation of the intercostal muscles results in changes in the mechanics of the lungs and thorax, such as a reduction in lung compliance and a strong restrictive ventilatory impairment. ${ }^{1}$ In individuals with tetraplegia inspiratory

*Correspondence: MTE Hopman, Department of Physiology, P.O. Box 9101, 6500 HB Nijmegen, The Netherlands force is about $60 \%$ compared to that of age-matched normal subjects. ${ }^{5}$ The efficiency of the unaffected muscles may be decreased due to instability of the chestwall and due to an inactive lifestyle. ${ }^{5}$ The respiratory muscle dysfunction in tetraplegics can be described as 'muscle weakness' (low maximal strength) and as 'muscle fatigue' (endurance capacity) of the respiratory muscles. ${ }^{6}$ Both appear to be significantly reduced in individuals with tetraplegia compared to able bodied individuals. ${ }^{7}$ The inspiratory endurance capacity of the respiratory muscles $\left(\mathrm{P}_{\text {endu }}\right)$ expressed as percentage of the maximal inspiratory strength of these muscles $\left(\mathrm{P}_{\text {imax }}\right)$ is about $50 \%$ in individuals with tetraplegia compared to about $80 \%$ in able bodied individuals. ${ }^{7}$ Limitation of exercise by muscle fatigue asks for training of the respiratory muscles. Training of these muscles may improve the daily activities. Respiratory muscle training in healthy subjects, COPD (Chronic Obstructive Pulmonary Disease) and other pulmonary patients has shown a positive effect. ${ }^{8-14}$ In individuals with tetraplegia training of the inspiratory 
muscles has been applied by Gross et $a l^{15}$ BieringSorensen et $a l^{16}$ and Derrickson et al. ${ }^{17}$ The latter compared inspiratory muscle training using inspiratory resistance to training with abdominal weights in individuals with tetraplegia shortly after the injury, which is an unstable period. There was no significant difference in pulmonary function between the two training programs, but the pulmonary function did improve after training. Gross et $a l^{15}$ and BieringSorensen et $a l^{16}$ studied individuals with tetraplegia after the rehabilitation period and thus in a more or less stable condition. Both had little or no control of the training intensity during the training period. Gross et $a l^{15}$ found an increase in strength and in endurance, but the two were not correlated with each other. BieringSorensen $e a^{16}$ compared the peak flow, lung volumes and ventilation- and diffusion-capacity. Only the peak flow showed a significant increase after training. In both studies the imposed inspiratory resistance (ie training intensity) was not objectively adjusted during the training, which may influence the training effect. The purpose of this study is to determine whether target flow endurance training of the inspiratory muscles, using a well-controlled training intensity, ${ }^{18,19}$ increases the strength and endurance of these muscles in individuals with complete and incomplete tetraplegia and, in addition, to examine the effect of this training on maximal exercise performance.

\section{Methods}

\section{Subjects}

Ten individuals (eight male and two female) with tetraplegia participated in this study. Due to illness, not related to the study, one female subject dropped out of the study. The lesion levels of the nine participating subjects varied between $\mathrm{C} 3$ and $\mathrm{C} 7$ and existed for at least 2 years with a mean of 11.1 year and a range from $2-27$ years. The ages varied from $20-49$ years with a mean of 34.4 years of age (Table 1). Three subjects had a complete lesion (ASIA impairment scale: (A), three subjects had a complete motor and an incomplete sensory lesion (B) and three subjects had an incomplete motoric and sensory lesion (C and $\mathrm{D})$. In this last group part of the muscles below the lesion were still innervated: one subject (subject 4 in Table 1) still had $10 \%$ of his motoric function (C); one still had $50 \%$ of the muscle strength in the left side of his body (subject 3) (D); the third could move all limbs, but had a decreased muscle strength and overall condition (subject 5) (C). Subjects with pulmonary disease or trauma of the chest wall were excluded from this study. The study was approved by the Faculty Ethical Committee and all subjects gave their written informed consent.

\section{Protocol}

On the first visit the subjects performed a series of tests including lung function tests and an exercise test. Following this, the subjects did a 'sham' training for 6 weeks with an incentive flow meter with no appreciable resistance. They were told that this training aimed at improving mobility of the chest. After this training period the tests were repeated, and all subjects participated in a 'real' target flow training program for 6 weeks with an incentive flow meter with a resistance. ${ }^{18,19}$ The subjects were used as their own control. Following the training the tests were repeated. During the training periods at 2 and 4 weeks, the subjects were tested at home to adjust the training intensity.

\section{Lung function tests}

All following tests were performed before training, after 6 weeks sham training and after 6 weeks real training. A lung function test was performed at the Department of Pulmonology Dekkerswald, University of Nijmegen, with a spirometer (Pulmonet 3, Sensormatics, Utrecht, The Netherlands). Slow Inspiratory Vital Capacity (IVC), Forced Expiratory Volume over

Table 1 Individual characteristics of the individuals with tetraplegia $(1-9)$ and individual results of the measurements at the start of the study

\begin{tabular}{|c|c|c|c|c|c|c|c|c|c|c|c|c|}
\hline Subject & Age & Gender & $\begin{array}{c}\text { Duration } \\
\text { of lesion } \\
\text { (years) }\end{array}$ & $\begin{array}{l}\text { Lesion } \\
\text { level/ } \\
\text { ASIA }\end{array}$ & $\begin{array}{c}\text { Motor } \\
\text { complete }\end{array}$ & $\begin{array}{c}\text { Sensor } \\
\text { complete }\end{array}$ & $\begin{array}{c}I V C \\
(l)\end{array}$ & $\begin{array}{l}P_{\text {imax }} \\
(\mathrm{kPa})\end{array}$ & $\begin{array}{c}P_{\text {endu }} \\
(k P a)\end{array}$ & $\begin{array}{l}R P_{\text {endu }} \\
(k P a)\end{array}$ & $\begin{array}{c}\dot{\mathrm{VO}} \mathrm{O}_{2} \\
\text { peak } \\
(\mathrm{l} / \mathrm{min})\end{array}$ & $\begin{array}{c}\text { POpeak } \\
(W)\end{array}$ \\
\hline 1 & 34 & $\mathrm{~m}$ & 15 & $\mathrm{C} 5 / \mathrm{A}$ & + & + & 2.15 & 8.41 & 4.20 & 50.0 & 0.44 & 18 \\
\hline 2 & 49 & $\mathrm{f}$ & 27 & $\mathrm{C} 7 / \mathrm{A}$ & + & + & 2.20 & 3.86 & 2.00 & 51.7 & 0.57 & 23 \\
\hline 3 & 44 & $\mathrm{~m}$ & 19 & $\mathrm{C} 5 / \mathrm{D}$ & - & - & 5.15 & 12.97 & 4.81 & 37.1 & 1.42 & 76 \\
\hline 4 & 41 & $\mathrm{~m}$ & 11 & $\mathrm{C} 6 / \mathrm{C}$ & - & - & 4.95 & 9.38 & 4.62 & 49.2 & 1.14 & 45 \\
\hline 5 & 36 & $\mathrm{~m}$ & 6 & $\mathrm{C} 3 / \mathrm{C}$ & - & - & 4.65 & 7.05 & 2.73 & 38.7 & 1.21 & 60 \\
\hline 6 & 35 & $\mathrm{~m}$ & 6 & C5/A & + & + & 3.05 & 9.52 & 6.72 & 70.6 & 0.55 & 9 \\
\hline 7 & 20 & $\mathrm{~m}$ & 11 & C6/B & + & - & 3.40 & 7.38 & 3.98 & 53.8 & 0.85 & 21 \\
\hline 8 & 26 & $\mathrm{~m}$ & 2 & C6/B & + & - & 3.50 & 8.68 & 4.06 & 46.7 & 1.01 & 43 \\
\hline 9 & 25 & $\mathrm{~m}$ & 3 & C6/B & + & - & 3.85 & 7.81 & 2.59 & 33.2 & 0.91 & 33 \\
\hline
\end{tabular}

$\mathrm{IVC}=$ Inspiratory Vital Capacity; $\mathrm{P}_{\text {imax }}=$ Maximal Inspiratory Pressure; $\mathrm{P}_{\mathrm{endu}}=$ Endurance Pressure; $\mathrm{RP} \mathrm{P}_{\mathrm{endu}}=$ Ratio between $\mathrm{P}_{\text {endu }}$ and $\mathrm{P}_{\text {imax }} ; \mathrm{VO}_{2}$ peak = peak oxygen consumption; POpeak= peak power output 
$1 \mathrm{~s}\left(\mathrm{FEV}_{1}\right)$ and Forced Inspiratory Volume over $1 \mathrm{~s}$ $\left(\mathrm{FIV}_{1}\right)$ were measured.

The respiratory muscles were tested with a static maximal mouth pressure test and an incremental threshold loading test. The static maximal mouth pressure test was used to assess the maximal inspiratory strength, ie maximal inspiratory mouth pressure $\left(\mathrm{P}_{\text {imax }}\right){ }^{20,21}$ The test was performed with a plastic flanged mouthpiece which was connected to a closed, rigid, plastic tube with a small leak (I.D. $1.8 \mathrm{~mm}$; length $40 \mathrm{~mm}$ ), to prevent buccal muscles from producing significant pressures, and to prevent closing of the glottis. ${ }^{22,23}$ A pressure transducer (Gould P23ID, Statham, USA), connected to a pressure monitor (Gould SP1405, Statham, USA) measured the pressure inside the tube. The results were recorded on a chart-recorder (Kipp \& Zonen BD41, Delft, The Netherlands). The pressure system was calibrated before each measurement with a mercury column. The subjects were in a comfortable sitting position, wearing a noseclip. The $\mathrm{P}_{\text {imax }}$ was measured at Residual Volume (RV). The maximal pressures had to be maintained for at least $1 \mathrm{~s}$, in order to reach a plateau. Each subject repeated the manoeuvre at least three times until two almost identical readings were obtained. The highest plateau-pressure value was used for analyses as being the maximal mouth pressure. All subjects were verbally encouraged to perform maximally and recieved visual feedback from the chart-recorder.

The incremental threshold loading test assessed the endurance capacity or fatiguability of the respiratory muscles. ${ }^{24}$ A modified weighted inspiratory valve was used. Weights could be added externally every minute without disrupting the system or breathing pattern. This system was connected to the pressure transducer and the chart-recorder. All subjects were in a comfortable sitting position, wearing a noseclip. The test consisted of a continuous incremental protocol in which weights were added every minute, varying between 10 and $40 \mathrm{~g}$, aiming at achieving maximal endurance capacity within $20 \mathrm{~min}$. The subjects were verbally encouraged to breath through the mouthpiece as long as possible. The highest pressure achieved, maintained for $1 \mathrm{~min}$, was defined as the $\mathrm{P}_{\text {endu }}$. The relative $\mathrm{P}_{\text {endu }}\left(\mathrm{RP}_{\text {endu }}\right)$ was calculated by expressing the $\mathrm{P}_{\text {endu }}$ as a percentage of the $\mathrm{P}_{\text {imax }}$.

\section{Exercise test}

The exercise test, performed at 0, 6 and 12 weeks, was an incremental maximal arm-cranking exercise test, performed on an electrically braked arm ergometer (modified cycle ergometer, Lode RS 232, Groningen, The Netherlands). If necessary the hands were fixed to the handles with especially designed mitts and the body was fixed to the wheelchair with an elastic belt. During the test ECG was monitored continuously. The expired air was analyzed using an automatic mixing chamber ergospirometer (Oxycon IV, Mijnhardt, Bunnik, The
Netherlands). Besides the peak power output (PO peak), the peak oxygen consumption ( $\dot{\mathrm{VO}}_{2}$ peak), the peak expiratory minute ventilation $\left(\mathrm{V}_{\mathrm{E}}\right.$ peak), the respiratory gas exchange value ( $R$-value), the breathing frequency and the tidal volume were measured. The ergospirometer was calibrated before each test with known gas mixtures. The cranking protocol was a continuously incremental protocol of $3-6 \mathrm{~W}$ per min, depending on the fitness level of the subject, starting at $3 \mathrm{~W}$. The protocol was the same for each subject the first, second and third time they performed the test. Each subject was verbally encouraged during the whole test. The test was terminated when the cycling frequency dropped below $40 \mathrm{rpm}$ or when the subject stopped for another reason.

\section{Training}

The subjects trained twice a day for 15 min during 6 weeks. The training consisted of inspiration through a mouthpiece connected to an incentive flow meter (INSPIRx Incentive Spirometer, Intertech, Sims, USA). A breathing pattern of $3 \mathrm{~s}$ inspiration and $4 \mathrm{~s}$ expiration was imposed. The subjects wore a noseclip. During the sham training the apparatus had no appreciable resistance. The subjects were unaware of this and thought they were performing a training of the chest mobility. During the real training the apparatus was fitted with an extra resistance of $2 \mathrm{~mm}$. The leak in the incentive flow meter was adjusted in such a way that the inspiratory mouth pressure was $70 \%$ of $\mathrm{P}_{\text {endu. }}{ }^{18}$

\section{Home test}

After 2 and 4 weeks of sham training the difference in chest circumference between maximal inspiration and maximal expiration was measured with a measuring tape, in accordance with the suggestion of improvement of the chest mobility. After 2 and 4 weeks of real training $\mathrm{P}_{\text {imax }}$ and $\mathrm{P}_{\text {endu }}$ were measured at the subjects home. If necessary, the training apparatus was readjusted to $70 \%$ of the newly achieved $\mathrm{P}_{\text {endu }}$.

\section{Statistical analysis}

The results of the different tests were analyzed with a paired Student $t$-test $(P<0.05)$

\section{Results}

The individual characteristics of the subjects in this study are shown in Table 1.

The IVC, FIV 1 and $\mathrm{FEV}_{1}$ showed no significant differences before and after either sham or real training.

After the sham training the $\mathrm{P}_{\text {endu }}$ was increased from 3.98 to $4.71 \mathrm{kPa}$ with a $P$-value of 0.05 . The sham training had no influence on the $P_{\text {imax }}$ and $\mathrm{RP}_{\text {endu }}$. 
The real training had no influence on the $\mathrm{P}_{\text {imax }}$. The increased significantly after the real training, from $4.71 \pm 1.49$ to $6.16 \pm 1.57 \mathrm{kPa}$ with a $P$-value of 0.01 and the $\mathrm{RP}_{\text {endu }}$ increased significantly from $58.3 \pm 18.6$ to $77.3 \pm 23.3 \%$ with a $P$-value of 0.02 . (Figure 1 )

The sham training had no effect on exercise performance. After the real training there was a tendency to an increase in PO peak (from $37.0 \pm 26.2 \mathrm{~W}$ before to $40.8 \pm 28.0 \mathrm{~W}$ after training), and $\mathrm{V}_{\mathrm{E}}$ peak (from $34.8 \pm 11.31 / \mathrm{min}$ before to $37.3 \pm 13.51 / \mathrm{min}$ after training). $\dot{\mathrm{VO}}_{2}$ peak increased significantly from $0.87 \pm 0.49$ to $0.97 \pm 0.441 / \mathrm{min}$ with a $P$-value of 0.05 . (Figure 2)

\section{Discussion}

\section{Lung function tests}

The IVC and $\mathrm{FEV}_{1}$ in the present study are lower in tetraplegic than in able bodied individuals ${ }^{7}$ (3.7 and 4.41 for IVC, 3.2 and 3.61 for $\mathrm{FEV}_{1}$, respectively), which is in agreement with previously reported results. $^{1-4}$ The mean values for $\operatorname{IVC}(3.71)$ and $\mathrm{FEV}_{1}$ (3.2 1) are higher than the values for individuals with tetraplegia in the study of Biering-Sorensen et $a l^{16}(2.4$ and 2.21 , respectively). This is probably due to the fact that in the present study individuals with incomplete lesions were included, in contrast to the study of Biering-Sorensen et al. ${ }^{16}$ Both, the present study and the study of Biering-Sorensen et $a l,{ }^{16}$ revealed no changes in IVC and $\mathrm{FEV}_{1}$ after training of the

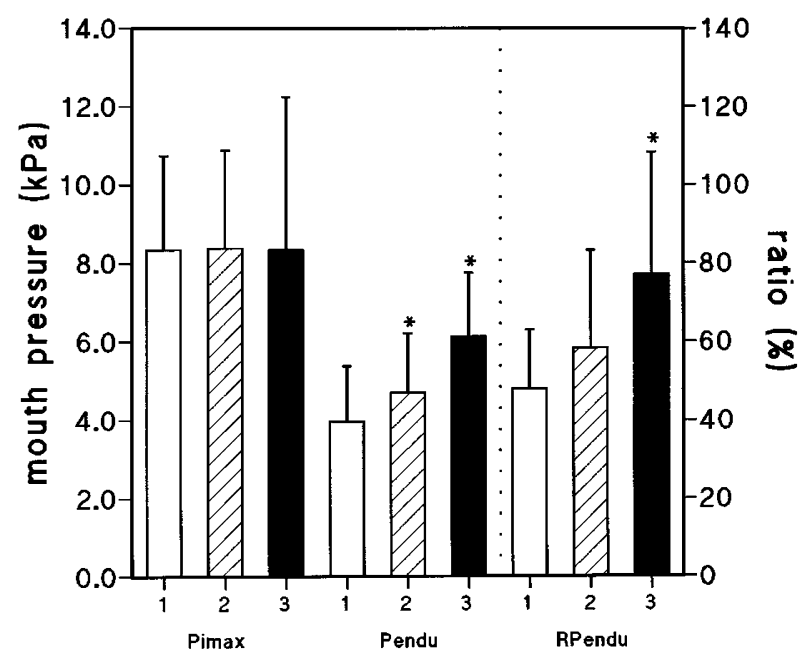

Figure 1 Lung function test: respiratory muscle strength and endurance. Maximal Inspiratory Pressure $\left(\mathrm{P}_{\text {imax }}\right)$, Endurance Pressure $\left(\mathrm{P}_{\text {endu }}\right)$ and Ratio between $\mathrm{P}_{\text {endu }}$ and $\mathrm{P}_{\text {imax }}\left(\mathrm{RP}_{\text {endu }}\right)$ before training (1), after 6 weeks sham training (2) and after 6 weeks real training (3) $* P<0.05$ inspiratory muscles. This suggests that the decrease in lung volume in individuals with tetraplegia does not depend on the strength and endurance of the remaining inspiratory muscles, but on changes in the mechanical behaviour of the lungs, such as a reduction in lung compliance and a strong ventilatory impairment. ${ }^{1}$

The mean value for the $\mathrm{P}_{\text {imax }}$ in the present study $(8.37 \mathrm{kPa})$ was higher than the values in the study of Hopman et $a l^{7}(5.91 \mathrm{kPa})$. Again this could be explained by the participation of subjects with incomplete lesions in our study in contrast to the study of Hopman et al. ${ }^{7} \mathrm{P}_{\text {endu }}$ and $\mathrm{RP}_{\text {endu }}$ before training (3.98 $\mathrm{kPa}$ and $47.9 \%$, respectively) are in agreement with the study of Hopman et $a l^{7}(2.71 \mathrm{kPa}$ and $49 \%$, respectively), these values are obviously less dependent on the completeness of the lesion. Comparing the values of the present study to values in able bodied individuals ${ }^{7}\left(\mathrm{P}_{\text {imax }} 8.37\right.$ and $8.41 \mathrm{kPa}$, respectively; $\mathrm{P}_{\text {endu }} 3.98$ and $6.91 \mathrm{kPa}$, respectively; $\mathrm{RP}_{\text {endu }}$ 47.9 and $82 \%$, respectively), lower values on endurance capacity are found in the spinal cord injured population, as expected, but the maximal inspiratory strength appears to be the same in both populations. This may indicate that the diaphragm and/or accessory inspiratory muscles play a pivotal role in $\mathrm{P}_{\text {imax }}$. The increase in endurance capacity of the inspiratory muscles after training (Figure 1) is in agreement with the results of Gross et $a l^{15}$ although they did find an increase in the strength of the inspiratory muscles as well. It is well known that the training-effect on muscles is specific to the training-mode. ${ }^{12}$ Since the

\section{Lung function test: respiratory muscle strength and endurance}

\section{muscles is specific to the training-mode. 2 Since the}

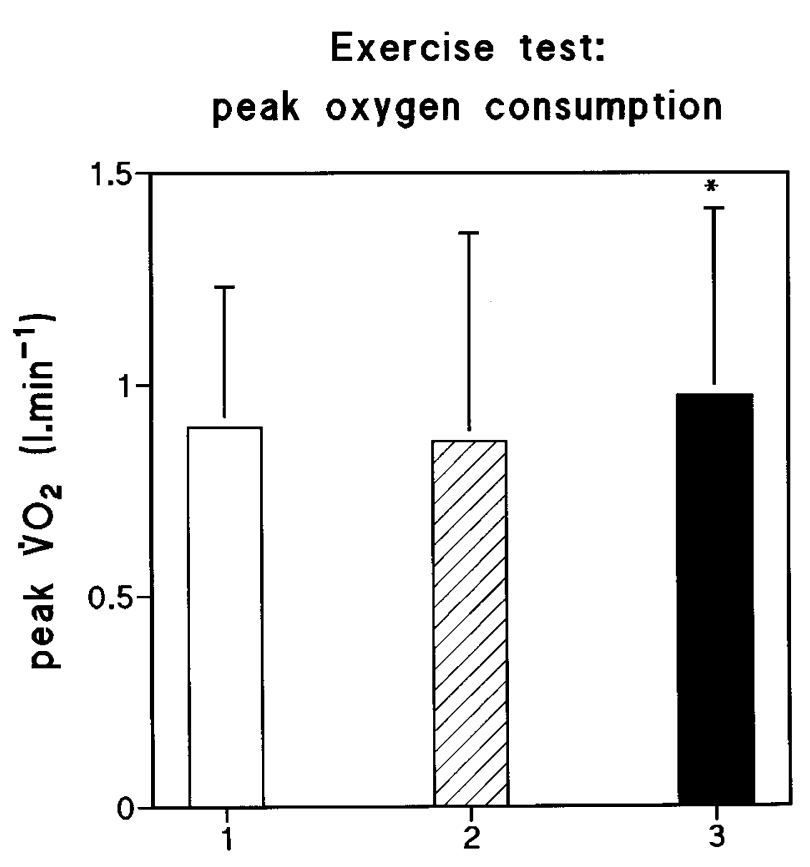

Figure 2 Exercise test: peak oxygen consumption. Peak oxygen consumption (peak $\mathrm{VO}_{2}$ ) before training (1), after 6 weeks sham training (2) and after 6 weeks real training (3). $* P<0.05$ 
training in the present study was a typical endurance training the improvement of the endurance capacity and the lack of improvement on strength of the respiratory muscles are in line with the trainingspecificity. The increase of $P_{\text {endu }}$ after the sham training (Figure 1) was not expected, the $P$-value being lower $(0.0457)$ than after the real training (0.0091). An explanation for the increase during the sham training could be due to the fact that the subjects where focused on their breathing and more aware of their breathing pattern.

\section{Exercise test}

The mean value for PO peak in the present study $(37.0 \mathrm{~W})$ was higher than the mean value for tetraplegic subjects in the study of Coutts et $a l^{25}$ $(24 \mathrm{~W})$, which may again be due to the participation of subjects with incomplete lesions in the present study. The mean values for the $\mathrm{VO}_{2}$ peak and the $\mathrm{V}_{\mathrm{E}}$ peak before training in this study $(0.87$ and $34.8 \mathrm{l} / \mathrm{min}$, respectively) were lower than Coutts' findings (0.97 and $501 / \mathrm{min}$, respectively). This could be explained by the participation of trained wheelchair athletes in the study of Coutts, while our study population was untrained. The increase in $\dot{\mathrm{VO}}_{2}$ peak, as was found after training, indicates an improvement in aerobic exercise performance (Figure 2). To our knowledge no earlier research has been carried out on exercise performance after respiratory muscle training in individuals with tetraplegia. The increase in $\dot{\mathrm{VO}}_{2}$ peak after training may be explained by an improvement in the oxygen uptake by either the respiratory muscles and/or the exercising arm muscles. The values for $\mathrm{VO}_{2}$ peak were still very small compared to the $\dot{\mathrm{VO}}_{2}$ peak for arm exercise in able bodied individuals, ${ }^{26}$ most likely due to the smaller active muscle-mass ${ }^{25}$ and the inactive lifestyle ${ }^{5}$ as mentioned before in the introduction.

\section{Conclusion}

Target flow endurance training of the inspiratory muscles in individuals with tetraplegia increases the endurance capacity of the inspiratory muscles, but has no measurable effect on the strength of these muscles. In addition, the results of this study show a better aerobic exercise performance of this population after training the inspiratory muscles.

\section{References}

1 De Troyer A, Heilporn A. Respiratory mechanics in quadriplegia. The respiratory function of the intercostal muscles. $\mathrm{Am}$ Rev Respir Dis 1980; 122: 591 - 600.

2 Forner JV. Lung volumes and mechanics of breathing in tetraplegics. Paraplegia 1980; 18: 258-266.
3 Hjeltnes N. Cardiorespiratory capacity in tetra- and paraplegia shortly after injury. Scan J Rehabil Med 1986; 18: 65-70.

4 Pentland B. Rehabilitation. Quadriplegia and cardiorespiratory fitness. The Lancet 1993; 341: $413-414$.

5 Grassino A. A rationale for training respiratory muscles. Int Rehabil Med 1984; 6: 175-178.

6 Respiratory Muscle Fatigue Workshop Group. NHLBI Workshop summary Respiratory muscle fatigue. Am Rev Respir Dis 1990; 142: $474-480$

7 Hopman MTE et al. Respiratory strength and endurance in individuals with tetraplegia. Spinal Cord 1997; 35: 104-108.

8 Akabas SR, Bazzy AR, DiMauro S, Haddad GG. Metabolic and functional adaptation of the diaphragm to training with resistive loads. J Appl Physiol 1989; 66: 529-535.

9 Belman MJ, Shadmehr R. Targeted resistive ventilatory muscle training in chronic obstructive pulmonary disease. $J$ Appl Physiol 1988; 65: $2726-2735$.

10 Boutellier U, Büchel R, Kundert A, Spengler C. The respiratory system as an exercise limiting factor in normal trained subjects. Eur J Appl Physiol 1992; 65: 347-353.

11 Grassino A. Inspiratory muscle training in COPD patients. Eur Respir J Suppl 1989; 7: 581s-586s.

12 Leith DE, Bradley M. Ventilatory muscle strength and endurance training. J Appl Physiol 1976; 41: 508-516.

13 Morgan DW, Kohrt WM, Bates BJ, Skinner JS. Effects of respiratory muscle endurance training on ventilatory and endurance performance of moderately trained cyclists. Int $J$ Sports Med 1987; 8: $88-93$.

14 Noseda A et al. Resistive inspiratory muscle training and exercise performance in COPD patients. A comperative study with conventional breathing retraining. Bull Eur Physiopathol Respir 1987; 23: $457-463$.

15 Gross D et al. The effect of training on strength and endurance of the diaphragm in quadriplegia. Am J Med 1980; 68: 27-35.

16 Biering-Sorensen F, Lehmann Knudsen J, Schmidt A, Bundgaard A, Christensen I. Effect of respiratory training with a mouth-nose-mask in tetraplegics. Paraplegia 1991; 29: 113-119.

17 Derrickson J, Ciesla N, Simpson N, Imle PC. A comparison of two breathing exercise programs for patients with quadriplegia. Physical Therapy 1992; 72: 763-769.

18 Dekhuijzen PNR, Folgering H, van Herwaarden C. Target-flow inspiratory muscle training during pulmonary rehabilitation in patients with COPD. Chest 1991; 99: 128-133.

19 Heydra Y, Dekhuijzen P, van Herwaarden C, Folgering H. Target flow inspiratory muscle training improves nocturnal saturation in patients with COPD. AM J Respir Crit Care Med 1996; 153: $260-265$

20 Black LF, Hyatt RE. Maximal respiratory pressures: normal values and relationship to age and sex. Am Rev Respir Dis 1969; 99: $696-702$.

21 Karvonen J, Saarelainen S, Nieminen MM. Measurement of respiratory muscle forces based on maximal inspiratory and expiratory pressures. Respiration 1994; 61: $28-31$.

22 Koulouris $\mathrm{N}$ et al. Comparison of two different mouthpieces for measurement of Pimax and Pemax in normal and weak subjects. Eur Respir J 1988; 1: 863-867.

23 Mayos M, Giner J, Casan P, Sanchis J. Measurement of maximal static respiratory muscle fatigue. Chest 1991; 100: $364-366$.

24 Martyn JB, Moreno RH, Pare PD, Pardy RL. Measurement of inspiratory muscle performance with incremental threshold loading. Am Rev Respir Dis 1987; 135: 919-923.

25 Coutts KD, Rhodes EC, McKenzie DC. Maximal exercise responses of tetraplegics and paraplegics. $J$ Appl Physiol: Respirat Environ Exercise Physiol 1983; 55: 479-482.

26 McArdle WD, Katch Fl, Katch VL. In: Exercise Physiology. 3rd edn. Lea \& Feiger: Philadelphia/London 1991, pp 330-340. 